Article

\title{
Edge Version of Metric Dimension and Doubly Resolving Sets of the Necklace Graph
}

\author{
Jia-Bao Liu ${ }^{1}$, Zohaib Zahid ${ }^{2}$, Ruby Nasir ${ }^{2}$ and Waqas Nazeer ${ }^{3, *}$ \\ 1 School of Mathematics and Physics, Anhui Jianzhu University, Hefei 230601, China; liujiabaoad@163.com \\ 2 Department of Mathematics, University of Management and Technology, Lahore 54000, Pakistan; \\ zohaib_zahid@hotmail.com (Z.Z.); rubynasir76@gmail.com (R.N.) \\ 3 Division of Science and Technology, University of Education, Lahore 54000, Pakistan \\ * Correspondence: nazeer.waqas@ue.edu.pk; Tel.: +9-232-1470-7379
}

Received: 23 September 2018; Accepted: 29 October 2018; Published: 7 November 2018

\begin{abstract}
Consider an undirected and connected graph $G=\left(V_{G}, E_{G}\right)$, where $V_{G}$ and $E_{G}$ represent the set of vertices and the set of edges respectively. The concept of edge version of metric dimension and doubly resolving sets is based on the distances of edges in a graph. In this paper, we find the edge version of metric dimension and doubly resolving sets for the necklace graph.
\end{abstract}

Keywords: necklace graph; resolving sets; edge version of metric dimension; edge version of doubly resolving sets

\section{Introduction and Preliminaries}

Let $G$ be a connected, simple and undirected graph consisting of nonempty finite sets $V_{G}$ of vertices and $E_{G}$ of edges. The order of a graph $G$ is $\left|V_{G}\right|$ and $\left|E_{G}\right|$ is the size of $G$. The number of vertices joining to $v$, where $v \in V_{G}$ is called a degree of that vertex and written as $d_{v} . \Delta(G)$ is the maximum degree in a graph $G$.

For any two vertices $x, y \in V_{G}$, the distance $d(x, y)$ is the length of a shortest path between $x$ and $y$. Let $R=\left\{r_{1}, r_{2}, \ldots, r_{l}\right\} \subset V_{G}$ be an ordered set and let $x \in V_{G}$, then $r(x, R)$ representation of $x$ with respect to $R$ is the $l$-tuple $\left(d\left(x, r_{1}\right), d\left(x, r_{2}\right), \ldots, d\left(x, r_{l}\right)\right) . R$ is said to be a resolving set if different vertices of $G$ have different representations with respect to $R$. The minimum number of vertices in a resolving set is called a basis for $G$ and the cardinality of the basis is known as the metric dimension of $G$, represented by $\operatorname{dim}(G)$. For $R=\left\{r_{1}, r_{2}, \ldots, r_{l}\right\} \subset V_{G}$, the $i$ th component of $r(x, R)$ is 0 if and only if $x=r_{i}$. Hence, to prove that $R$ is a resolving set it is enough to show that $r(x, R) \neq r(y, R)$ for each pair $x \neq y \in V_{G} \backslash R$.

The following lemma is very helpful for determining resolving set for $\operatorname{dim}(G)$ :

Lemma 1. Let $R$ be a resolving set for a simple connected graph $G$ and $x, y \in V_{G}$. If $d(x, r)=d(y, r)$ for all vertices $r \in V_{G} \backslash\{x, y\}$, then $\{x, y\} \cap R \neq \varnothing$.

The idea of resolving sets and metric dimension was presented by Slater in [1] and also by Hararay and Melter in [2]. Metric dimension is applied in different branches of navigation [3], robotics [3], chemistry [4], and network discovery and verification. It is well-known in [5] that computing the metric dimension of a graph is an NP-hard problem. Metric dimension has been deeply elaborated in surveys $[6,7]$. The line graph $L(G)$ of a graph $G$ is defined as, the graph whose vertices are the edges of $G$, with two adjacent vertices if the corresponding edges share the common vertex in $G$. Also, metric properties of line graphs were studied to a great extent in [8-18]. The line graph of a graph $G$ is helpful to find edge distances using the same technique of finding vertex distances of the graph $G$. 
The concept of edge metric dimension was set up by Kelenc, Tratnik and Yero in [19] in 2016. They computed the edge metric dimension of different families of graphs and showed edge metric dimension i.e., $\operatorname{edim}(G)$ can be less, equal to or more than $\operatorname{dim}(G)$. They also showed computing $\operatorname{edim}(G)$ is NP-hard in general. Since in literature edge metric dimension exists and that is entirely different from edge metric dimension defined in [20], so we renamed edge metric dimension proposed in [20] as an edge version of metric dimension. The edge version of metric dimension is defined as:

\section{Definition 1.}

1. The edge distance $d_{E}(f, g)$ between two edges $f, g \in E_{G}$ is the length of a shortest path between vertices $f$ and $g$ in the line graph $L(G)$.

2. If $d_{E}(e, f) \neq d_{E}(e, g)$, then the edge $e \in E_{G}$ is said to resolve two edges $f$ and $g$ of $E_{G}$.

3. Suppose that $R_{E}=\left\{f_{1}, f_{2}, \ldots, f_{k}\right\} \subset E_{G}$ is an ordered set and $e$ is an edge of $E_{G}$, then $r_{E}\left(e, R_{E}\right)$ the edge version of representation of e with respect to $R_{E}$ is the $k$-tuple $\left(d_{E}\left(e, f_{1}\right), d_{E}\left(e, f_{2}\right), \ldots, d_{E}\left(e, f_{k}\right)\right)$.

4. If different edges of $G$ have different edge version of representations with respect to $R_{E}$, then the set $R_{E}$ is said to be a an edge version of resolving set of $G$.

5. The edge version of the metric basis of $G$ is basically an edge version of the resolving set having minimum cardinality. The cardinality of the edge version of metric basis is represented by $\operatorname{dim}_{E}(G)$, and is called the edge version of metric dimension of $G$.

In literature the edge version of metric dimension is known for few classes of graphs. Bounds of an edge version of metric dimension are also known and these bounds are given in the next theorem:

Theorem 1 ([21]). If $G$ is a connected graph with $\left|V_{G}\right| \geq 5$, then

$$
\left\lceil\log _{2} \Delta(G)\right\rceil \leq \operatorname{dim}_{E}(G) \leq\left|V_{G}\right|-2
$$

Table 1, represents all those graphs for which the edge version of metric dimension is known. In the table $P_{n}, C_{n}$ and $K_{n}$ represent the path graph, the cycle graph and the complete graph on $n$ vertices respectively. $W_{1, n}=K_{1}+C_{n}$ is a wheel graph on $n+1$ vertices, $K_{s, t}$ is a complete bipartite graph on $s+t$ vertices and for $n \geq 2, B_{n}=\left(k_{1}, k_{2}, \ldots, k_{n}\right)$ is a bouquet of circles $C^{1}, C^{2}, \ldots, C^{n}$ with a cut-vertex where $k_{i}$ is the number of vertices of $C^{i}(1 \leq i \leq n)$. Also $S_{n}$ represents the $n$-sunlet graph and $D_{n}$ is the prism graph on $2 n$ vertices.

Table 1. Edge version of the metric dimension of graphs.

\begin{tabular}{|c|c|}
\hline $\begin{array}{l}G \\
P_{n} \\
C_{n} \\
K_{n}\end{array}$ & $\begin{array}{l}\operatorname{dim}_{E} G \\
1[4] \\
\quad 2 \\
n-1[6]\end{array}$ \\
\hline$K_{s, t}$ & $\begin{array}{ll}\left\lfloor\frac{2(s+t-1)}{3}\right\rfloor & \text { if } s \leq t \leq 2 s \\
t-1 & \text { if } t \geq 2 s\end{array}$ \\
\hline$W_{1, n}$ & $\begin{cases}3 & \text { if } n=3,4 \\
4 & \text { if } n=5 \\
n-\left\lceil\frac{n}{3}\right\rceil & \text { if } n \geq 6\end{cases}$ \\
\hline$B_{n}$ & $2 n-1[23]$ \\
\hline$S_{n}$ & $\left\{\begin{array}{l}2 \text { if } n \text { is even; } \\
3 \text { if } n \text { is odd. }\end{array}\right.$ \\
\hline$D_{n}$ & $3[20]$ \\
\hline
\end{tabular}


Caceres et al. define the notion of a doubly resolving set in [22]. The doubly resolving sets present a valuable source for finding upper bounds of the metric dimension of graphs. Let the vertices $a$ and $b$ of the graph $G$ with order $\left|V_{G}\right| \geq 2$ doubly resolve vertices $c$ and $d$ of the graph $G$ if $d(c, a)-d(c, b) \neq d(d, a)-d(d, b)$. A subset $D$ of vertices doubly resolves $G$ if every two vertices in $G$ are doubly resolved by some two vertices of $D$. Moreover, in $G$ there do not exist any two different vertices having the same difference between their corresponding metric coordinates with respect to $D$. A doubly resolving set with minimum cardinality is called the minimal doubly resolving set. The minimum cardinality of a doubly resolving set for $G$ is represented by $\psi(G)$. In case of some convex prism, hamming and polytopes graphs, the minimal doubly resolving sets have been obtained in [24-26] respectively.

Clearly, every doubly resolving set is a resolving set, which implies $\operatorname{dim}(G) \leq \psi(G)$ for all graphs G. Also, if $a$ and $b$ doubly resolve $c$ and $d$, then $d(c, a)-d(d, a) \neq 0$ or $d(f, b)-d(g, b) \neq 0$, and thus $a$ or $b$ resolve $c$ and $d$, this shows that a doubly resolving set is also a resolving set.

Ahmed et al. in [27] proposed the idea of minimal edge version of doubly resolving sets of graph $G$, based on the distances of the edges of graph $G$ which is defined as follows:

\section{Definition 2.}

1. The edges $f$ and $g$ of the graph $G$ with size $\left|E_{G}\right| \geq 2$ are supposed to edge doubly resolve edges $f_{1}$ and $f_{2}$ of the graph $G$ if $d_{E}\left(f_{1}, f\right)-d_{E}\left(f_{1}, g\right) \neq d_{E}\left(f_{2}, f\right)-d_{E}\left(f_{2}, g\right)$ in $G$.

2. Let $D_{E}=\left\{e_{1}, e_{2}, \ldots, e_{k}\right\}$ be an ordered set of the edges of $G$. If any two edges $e \neq f \in E_{G}$ are doubly resolved by any two edges of set $D_{E}$ in $G$, then the set $D_{E} \subset E_{G}$ is said to be an edge version of doubly resolving set of $G$. The minimum cardinality of an edge version of doubly resolving set of $G$ is represented by $\psi_{E}(G)$.

Note that every edge version of a doubly resolving set is an edge version of a resolving set,

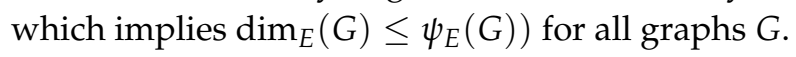

In this paper we compute the edge version of metric dimension and doubly resolving set for the necklace graph. At the end, we conclude that edge version of metric dimension and doubly resolving set are independent of choice of $n$.

\section{The Edge Version of Metric Dimension for $N_{e_{n}}$}

The necklace graph (see Figure 1) denoted by $N_{e_{n}}$ [28] is a cubic Halin graph [29] obtained by joining a cycle with all vertices of degree 1 of a caterpillar (also called a comb) having $n$ vertices of degree 3 and $n+2$ vertices of degree 1 , denoted by $x_{0}, x_{1}, \ldots, x_{n+1}$ and $y_{1}, y_{2}, \ldots, y_{n}$, respectively. We have $V_{N_{e_{n}}}=\left\{x_{0}, \ldots, x_{n+1}, y_{1}, \ldots, y_{n}\right\}$ and $E_{N_{e_{n}}}=F \cup H \cup K$, where $F=\left\{f_{1}, f_{2}, \ldots, f_{n+1}\right\}$, $H=\left\{h_{1}, h_{2}, \ldots, h_{n+1}\right\}$ and $K=\left\{g_{1}, g_{2}, \ldots, g_{n+1}\right\}$. The necklace graph is 3-regular graph with constant metric dimension, which is computed in [30] given below:

$$
\operatorname{dim}\left(N_{e_{n}}\right)= \begin{cases}2 & \text { if } n \text { is even } \\ 3 & \text { if } n \text { is odd }\end{cases}
$$




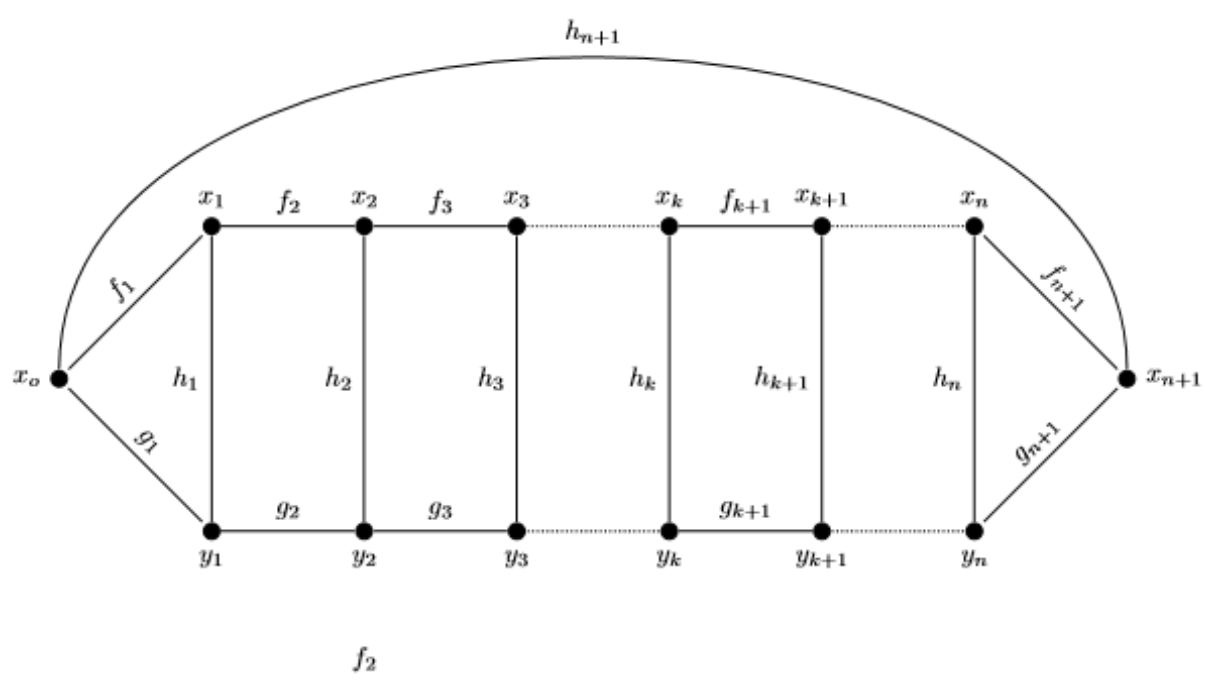

Figure 1. The necklace graph $N_{e_{n}}$.

For the edge version of metric dimension of the necklace graph, we have to construct a line graph $L\left(N_{e_{n}}\right)$ of $N_{e_{n}}$ with $n \geq 2$. (see Figure 2).

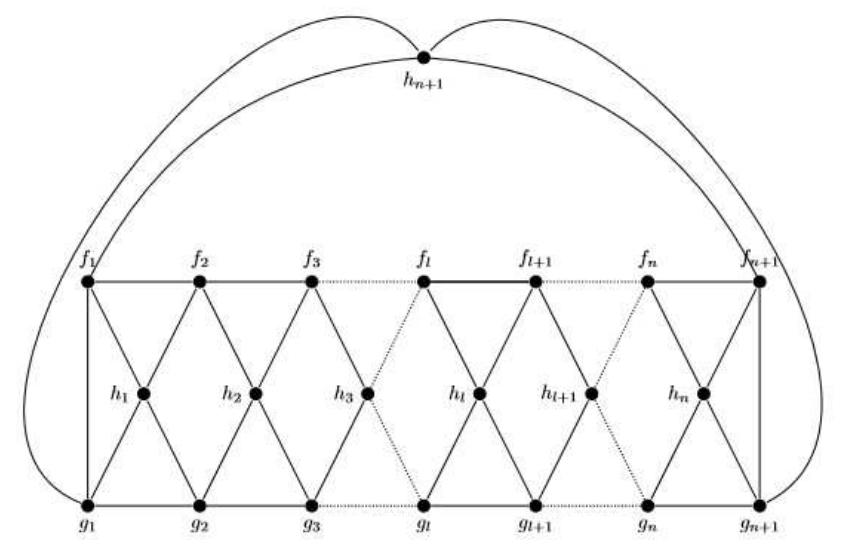

Figure 2. The line graph of a necklace graph: $L\left(N_{e_{n}}\right)$.

Theorem 2. The edge version of metric dimension of $N_{e_{n}}$ is 3 for $n \geq 2$.

Proof. Let $l=\left\lfloor\frac{n}{2}\right\rfloor$. For $n=2$ and 3, consider the set $R_{E}=\left\{f_{1}, f_{n}, f_{n+1}\right\} \subset E_{N_{e_{n}}}$, then the edge version of representation of each edge of $N_{e_{n}}$ with respect to $R_{E}$ is given below:

The edge version of representation of the edges $f_{p} \in F$ with respect to $R_{E}$ is:

$$
r_{E}\left(f_{p}, R_{E}\right)= \begin{cases}(0, n-1,2) & \text { if } p=1 \\ (p-1,|n-p|, n-p+1) & \text { if } 2 \leq p \leq n \\ (2, l, 0) & \text { if } p=n+1\end{cases}
$$


The edge version of representation of the inner edges $h_{p} \in H$ with respect to $R_{E}$ is:

$$
r_{E}\left(h_{p}, R_{E}\right)= \begin{cases}(p, n-p, n-p+1), & \text { if } 1 \leq p \leq n-1 \\ (n, 1,1), & \text { if } p=n \\ (1, l+1,1), & \text { if } p=n+1 .\end{cases}
$$

The edge version of representation of the edges $g_{p} \in K$ with respect respect to $R_{E}$ is:

$$
r_{E}\left(g_{p}, R_{E}\right)= \begin{cases}(1, n, 2), & \text { if } p=1 \\ (p, 2, n-p+2), & \text { if } 2 \leq p \leq n \\ (2, l+1,1), & \text { if } p=n+1\end{cases}
$$

For $n \geq 4$, take a set $R_{E}=\left\{f_{1}, f_{l}, f_{n}\right\} \subset E_{N_{e_{n}}}$, we will show that $R_{E}$ is an edge version of resolving set for $N_{e_{n}}$.

The edge version of representation of the edges $f_{p} \in F$ with respect to $R_{E}$ is:

$$
r_{E}\left(f_{p}, R_{E}\right)= \begin{cases}(p-1, l-p, p+2) & \text { if } 1 \leq p \leq l-1 \\ (p-1,|l-p|, n-p) & \text { if } l \leq p \leq l+2 \\ (n-p+3,|l-p|,|n-p|) & \text { if } l+3 \leq p \leq n \\ (2, l+1,1), & \text { if } p=n+1 .\end{cases}
$$

The edge version of representation of the inner edges $h_{p} \in H$ with respect to $R_{E}$ is:

$$
r_{E}\left(h_{p}, R_{E}\right)= \begin{cases}(p, l-p, p+3), & \text { if } 1 \leq p \leq l-2 ; \\ (p,|l-p|+1, n-p), & \text { if } l-1 \leq p \leq l+1 ; \\ (n-p+3,|l-p|+1, n-p), & \text { if } l+2 \leq p \leq n-1 ; \\ (3,|l-n|+1,1), & \text { if } p=n \\ (1, l, 2), & \text { if } p=n+1 .\end{cases}
$$

The edge version of representation of the edges $g_{p} \in K$ with respect respect to $R_{E}$ is:

$$
r_{E}\left(g_{p}, R_{E}\right)= \begin{cases}(p, l-p+1, p+2), & \text { if } 1 \leq p \leq l-1 ; \\ (p, 2, n-p+1), & \text { if } l \leq p \leq l+1 ; \\ (n-p+3, p-l+1, n-p+1), & \text { if } l+2 \leq p \leq n-1 ; \\ (3, n-l+1,2), & \text { if } p=n ; \\ (2, l+1,2), & \text { if } p=n+1 .\end{cases}
$$

From the above representations it is clear that no two edges of $N_{e_{n}}$ have the same edge version of representations, which implies $R_{E}$ is the edge version of resolving set and hence $\operatorname{dim}_{E}\left(N_{e_{n}}\right) \leq 3$. Next, we have to show that $\operatorname{dim}_{E}\left(N_{e_{n}}\right) \geq 3$. Suppose on the contrary that $\operatorname{dim}_{E}\left(N_{e_{n}}\right)=2$, then we have the following possibilities:

1. Let two edges $h_{1}$ and $h_{p}$ from the edge set $H$ with $2 \leq p \leq l+1$. For $R_{E}=\left\{h_{1}, h_{p}\right\} \subset E_{N_{e_{n}}}$, we have $r_{E}\left(f_{1}, R_{E}\right)=r_{E}\left(g_{1}, R_{E}\right)=(1, p)$, a contradiction. 
2. Now suppose that both edges are from the edge set $F$. Suppose without loss of generality that $R_{E}=\left\{f_{1}, f_{p}\right\} \subset E_{N_{e_{n}}}$, where $2 \leq p \leq l+1$. Then, $r_{E}\left(g_{1}, R_{E}\right)=r_{E}\left(h_{n+1}, R_{E}\right)=(1, p)$, a contradiction.

3. Now suppose that both edges are from the edge set $K$. Suppose without loss of generality that $R_{E}=\left\{g_{1}, g_{p}\right\} \subset E_{N_{e_{n}}}$, where $2 \leq p \leq l+1$. Since $r_{E}\left(f_{1}, R_{E}\right)=r_{E}\left(h_{n+1}, R_{E}\right)=(1, p)$, so we have a contradiction.

4. If one edge belongs to the set $F$ and the second edge is from $H$, without loss of generality we can take $R_{E}=\left\{f_{1}, h_{p}\right\} \subset E_{N_{e_{n}}}$ with $1 \leq p \leq l$. For $2 \leq n \leq 5$, we have $r_{E}\left(g_{n+1}, R_{E}\right)=$ $r_{E}\left(f_{n+1}, R_{E}\right)=(2,3)$ for $p=1$ and $r_{E}\left(g_{n+1}, R_{E}\right)=r_{E}\left(f_{n+1}, R_{E}\right)=(2, n-1)$ for $p=2$. For $n \geq 6$, we have $r_{E}\left(f_{n}, R_{E}\right)=r_{E}\left(g_{n}, R_{E}\right)=(3, p+3)$, a contradiction.

5. If one edge belongs to the set $G$ and the second edge is from $H$. i.e. $R_{E}=\left\{g_{1}, h_{p}\right\} \subset E_{N_{e_{n}}}$. This case is similar to (4).

6. If one edge belongs to the set $F$ and the second edge is from $K$, then we have the following five subcases:

(a) Let $R_{E}=\left\{f_{1}, g_{1}\right\} \subset E_{N_{e_{n}}}$. For $2 \leq n \leq 5$, we have $r_{E}\left(h_{1}, R_{E}\right)=r_{E}\left(h_{n+1}, R_{E}\right)=(1,1)$. For $n \geq 6$ and $l+4 \leq p \leq n+1$, we have $r_{E}\left(g_{p}, R_{E}\right)=r_{E}\left(f_{p}, R_{E}\right)=(n-p+3, n-p+3)$, a contradiction.

(b) If $R_{E}=\left\{f_{1}, g_{p}\right\} \subset E_{N_{e_{n}}}$ and $2 \leq p \leq l+1$, then we have $r_{E}\left(g_{1}, R_{E}\right)=r_{E}\left(h_{1}, R_{E}\right)=(1, p-1)$, a contradiction.

(c) Let $R_{E}=\left\{g_{1}, f_{p}\right\} \subset E_{N_{e_{n}}}$ with $2 \leq p \leq l+1$. This case is similar to 6 (b).

(d) Let $R_{E}=\left\{f_{2}, g_{p}\right\} \subset E_{N_{e_{n}}}$ with $1 \leq p \leq l$. For $2 \leq n \leq 5$ and $p=1$, we have $r\left(f_{1}, R_{E}\right)=$ $r_{E}\left(h_{1}, R_{E}\right)=(1,1)$ and when $p=2$, then $r_{E}\left(h_{1}, R_{E}\right)=r_{E}\left(h_{2}, R_{E}\right)=(1,1)$. For $n \geq 6$, we have $r_{E}\left(f_{n}, R_{E}\right)=r_{E}\left(h_{n}, R_{E}\right)=(4, p+2)$, a contradiction.

(e) Let $R_{E}=\left\{g_{2}, f_{p}\right\} \subset E_{N_{e_{n}}}$. This case is similar to 6 (e).

All the above possibilities lead to a contradiction. Hence, there is no edge version of resolving set of cardinality 2 for edges $E_{N_{e_{n}}}$, which implies that $\operatorname{dim}_{E}\left(N_{e_{n}}\right)=3$.

\section{The Minimal Edge Version of Doubly Resolving Sets for $N_{e_{n}}$}

The minimum doubly resolving set for the necklace graph $N_{e_{n}}$ has been discussed in [31]. In this section, we determine minimal edge version of doubly resolving sets for the necklace graph. Define $S_{j}\left(h_{n+1}\right)=\left\{g \in E_{N_{e_{n}}}: d_{E}\left(h_{n+1}, g\right)=j\right\}$ be the set of edges in $N_{e_{n}}$ at edge distance $j$ from edge $h_{n+1}$. The Table 2 can be easily formulated for $S_{j}\left(h_{n+1}\right)$ and it will be used to get the edge distances between two arbitrary edges in $E_{N_{e_{n}}}$.

Table 2. $S_{j}\left(h_{n+1}\right)$ for $N_{e_{n}}$.

\begin{tabular}{ccc}
\hline$n$ & $j$ & $S_{j}\left(h_{n+1}\right)$ \\
& 1 & $\left\{g_{1}, f_{1}, g_{n+1}, f_{n+1}\right\}$ \\
$2 t(t \geq 2)$ & $t+j \leq t$ & $\left\{g_{j}, f_{j}, g_{n+2-j}, f_{n+2-j}, h_{j-1}, h_{n+2-j}\right\}$ \\
$2 t+1(t \geq 2)$ & $t+1$ & $\left\{h_{t}, f_{t+1}, g_{t+1}\right\}$ \\
\hline
\end{tabular}

By the symmetry of the necklace graph $N_{e_{n}}$, it is clear that $d_{E}\left(f_{j}, f_{s}\right)=d_{E}\left(g_{j}, g_{s}\right)=$ $d_{E}\left(h_{n+1}, f_{s-j}\right)=d_{E}\left(h_{n+1}, g_{s-j}\right)$ for $s>j$

For $n=2 t$

$$
d_{E}\left(g_{j}, f_{s}\right)= \begin{cases}d_{E}\left(h_{n+1}, g_{|s-j|}\right)+1 & \text { if }|j-s| \leq t, 1 \leq j, s \leq n \\ d_{E}\left(h_{n+1}, g_{|s-j|}\right) & \text { if }|j-s|>t, 1 \leq j, s \leq n \\ d_{E}\left(h_{n+1}, g_{s}\right) & \text { if } j=s=1, \text { or } n+1 \\ d_{E}\left(h_{j}, g_{j+2}\right) & \text { if } 1<\mathrm{j}=\mathrm{s}<\mathrm{n} \\ d_{E}\left(h_{n+1}, f_{n}\right) & \text { if } j=s=n .\end{cases}
$$


For $n=2 t+1$

$$
d_{E}\left(g_{j}, f_{s}\right)= \begin{cases}d_{E}\left(h_{n+1}, g_{|s-j|}\right) & \text { if } s>j \\ d_{E}\left(h_{n+1}, g_{s}\right) & \text { if } j=s=1, \text { or } n+1 \\ d_{E}\left(h_{j}, g_{j+2}\right) & \text { if } 1<j=s<n \\ d_{E}\left(h_{n+1}, f_{n}\right) & \text { if } j=s=n\end{cases}
$$

Lemma 2. $\psi_{E}\left(N_{e_{n}}\right)=3$, whenever $n=2 t, t \geq 2$.

Proof. The Table 3 represents the vectors of edge version of representations of $N_{e_{n}}$ with respect to $D_{E}=\left\{f_{1}, f_{t+1}, f_{n+1}\right\}$

Table 3. Vectors of edge version of representations of $N_{e_{n}}, n=2 t$.

\begin{tabular}{ccc}
\hline$j$ & $S_{j}\left(h_{n+1}\right)$ & $D_{E}=\left\{f_{1}, f_{t+1}, f_{n+1}\right\}$ \\
0 & $h_{n+1}$ & $(1, t+1,1)$ \\
1 & $g_{1}$ & $(1, t+2,2)$ \\
& $f_{1}$ & $\{0, t, 2\}$ \\
& $g_{n+1}$ & $\{2, t, 0\}$ \\
$2 \leq j \leq k$ & $f_{n+1}$ & $\{j, t+2-j, j+1\}$ \\
& $g_{j}$ & $\{j, t+1-j, j+1\}$ \\
& $f_{j}$ & $\{n-j+3, j-t, n-j+2\}$ \\
& $g_{n+2-j}$ & $\{j, t+1-j, j+2\}$ \\
& $f_{n+2-j}$ & $\{n-j+3,|t+1-j|, n-j+1\}$ \\
& $h_{j-1}$ & $\{t, 0, t\}$ \\
& $h_{n+2-j}$ & $\{n-j+3, t-j, n-j+1\}$ \\
& $h_{t}$ & $\{t+1,2, t+1\}$ \\
& $h_{t+1}$ & \\
& $f_{k+1}$ &
\end{tabular}

It can be verified that for each $j \in\{1,2,3, \ldots, t+1\}$, no two edges $f, g \in S_{j}\left(h_{n+1}\right)$ exist such that $r_{E}\left(f, D_{E}\right)-r_{E}\left(g, D_{E}\right)=0$ holds. Also for any $j, s \in\{1,2,3, \ldots, t+1\}$, there do not exist any two edges $f \in S_{i}\left(w_{n+1}\right)$ and $g \in S_{j}\left(h_{n+1}\right)$ such that $r_{E}\left(f, D_{E}\right)-r_{E}\left(g, D_{E}\right)=i-j$. So, $D_{E}=\left\{f_{1}, f_{t+1}, f_{n+1}\right\}$ becomes the minimal edge version of doubly resolving set for $n=2 t, t \geq 2$ and therefore the Lemma 2 holds.

Lemma 3. $\psi_{E}\left(N_{e_{n}}\right)=3$ whenever, $n=2 t+1, t \geq 2$.

Proof. As we know that $\operatorname{dim}_{E}\left(N_{e_{n}}\right) \leq \psi_{E}\left(N_{e_{n}}\right)$ holds. Now the Table 4 represents the vectors of edge version of representations of $N_{e_{n}}$ with respect to $D_{E}=\left\{f_{1}, f_{n}, h_{t+1}\right\}$.

Table 4 shows that for $j \in\{1,2,3, \ldots, t+1\}$ there do not exist two edges $f, g \in S_{j}\left(h_{n+1}\right)$ such that the following condition $r_{E}\left(f, D_{E}\right)-r_{E}\left(g, D_{E}\right)=0$ holds. Also, for any $i, j \in\{1,2,3, \ldots, t+1\}$, there do not exist any two edges $f \in S_{i}\left(h_{n+1}\right)$ and $g \in S_{j}\left(h_{n+1}\right)$ such that $r_{E}\left(f, D_{E}\right)-r_{E}\left(g, D_{E}\right)=i-j$. So, $D_{E}=\left\{f_{1}, f_{n}, h_{t+1}\right\}$ becomes the minimal edge version of doubly resolving set for $n=2 t+1, t \geq 2$ and therefore the Lemma 3 holds. 
Table 4. Vectors of edge version of representations of $N_{e_{n}}$, for $n=2 t+1$.

\begin{tabular}{ccc}
\hline$j$ & $S_{j}\left(h_{n+1}\right)$ & $D_{E}=\left\{f_{1}, f_{n}, h_{t+1}\right\}$ \\
0 & $h_{n+1}$ & $(1,2, t+2)$ \\
1 & $g_{1}$ & $(1,3, t+1)$ \\
& $f_{1}$ & $\{0,3, t+1\}$ \\
& $g_{n+1}$ & $\{2,2, t+1\}$ \\
& $f_{n+1}$ & $\{2,1, t+1\}$ \\
2 & $g_{n}$ & $\{j+1,2, t+2-j\}$ \\
& $h_{n}$ & $\{j+1,1, t+3-j\}$ \\
$\leq j \leq t$ & $g_{j}$ & $\{j, j+2, t+2-j\}$ \\
& $f_{j}$ & $\{j, j+2, j\}$ \\
& $g_{n+1-j}$ & $\{j+1, j-1, t-j+2\}$ \\
& $f_{n+2-j}$ & $\{j+1, j-2, t-j+2\}$ \\
& $h_{j-1}$ & $\{j-1, j+2, j\}$ \\
& $h_{n+1-j}$ & $\{j+1, j-2, t-j+3\}$ \\
& $f_{t+1}$ & $\{t, t, 1\}$ \\
& $g_{t+1}$ & $\{t+1, t+1,1\}$ \\
& $f_{t+2}$ & $\{t+1, t-2,1\}$ \\
& $g_{t+2}$ & $\{t+2, t+1,1\}$ \\
& $h_{t+2}$ & $\{t+2, t-1,2\}$ \\
\hline & &
\end{tabular}

Note: A counting technique determines that $\psi_{E}\left(N_{e_{n}}\right)=3$ for $n=2$ and 3 . The sets $\left\{f_{1}, f_{2}, f_{3}\right\}$ and $\left\{f_{1}, f_{3}, h_{2}\right\}$ are the minimal edge version of doubly resolving sets for $N_{e_{2}}$ and $N_{e_{3}}$ respectively. When Lemma 2 and Lemma 3 is combined, then the following main theorem is formulated.

Theorem 3. Let $N_{e_{n}}$ be the necklace graph. Then $\psi_{E}\left(N_{e_{n}}\right)=3$ for $n \geq 2$.

\section{Conclusions}

In this paper, we have extended the notion of metric dimension to the edge version of metric dimension for the necklace graph $N_{e_{n}}$ which is the least cardinality over all edge versions of resolving sets. We also calculated the minimal edge version of doubly resolving sets for $N_{e_{n}}$. It is interesting to consider the necklace graph because its edge version of metric dimension and the minimal edge version of doubly resolving set are independent of parity of $n$. In previous work on necklace graphs, (see $[30,31]$ ) resolving sets were based on vertices and distances were calculated between vertices only. While, in this paper edges have been considered for getting resolving sets and distances have been calculated between edges. Finally, we get $\operatorname{dim}_{E}\left(N_{e_{n}}\right)=\psi_{E}\left(N_{e_{n}}\right)=3$ for every $n$.

Author Contributions: J.-B.L. designed the problem. Z.Z. investigate the problem. R.N. wrote the paper and W.N. validate the results.

Funding: This research was funded by China Postdoctoral Science Foundation under grant No. 2017M621579 and Postdoctoral Science Foundation of Jiangsu Province under grant No. 1701081B. Project of Anhui Jianzhu University under Grant no. 2016QD116 and 2017dc03. Anhui Province Key Laboratory of Intelligent Building \& Building Energy Saving.

Acknowledgments: The authors are grateful to the reviewers for suggestions to improve the presentation of the manuscript.

Conflicts of Interest: The authors declare no conflict of interest.

Open Problem: Characterize all classes of graphs in which metric dimension/doubly resolving sets coincides with edge version of metric dimension/doubly resolving sets. 


\section{References}

1. Slater, P.J. Leaves of trees. Congr. Numer. 1975, 14, 549-559.

2. Harary, F.; Melter, R.A. On the metric dimension of a graph. Ars Combin. 1976, 2, 191-195.

3. Khuller, S.; Raghavachari, B.; Rosenfeld, A. Landmarks in Graphs. Discret. Appl. Math. 1996, 70, $217-229$. [CrossRef]

4. Chartrand, G.; Eroh, L.; Johnson, M.A.; Oellermann, O.R. Resolvability in graphs and the metric dimension of a graph. Discret. Appl. Math. 2000, 105, 99-113. [CrossRef]

5. Garey, M.R.; Johnson, D.S. Computers and Intractability: A Guide to the Theory of NP-Completeness; Freeman: New York, NY, USA, 1979.

6. Bailey, R.F.; Cameron, P.J. Base size, metric dimension and other invariants of groups and graphs. Bull. Lond. Math. Soc. 2011, 43, 209-242. [CrossRef]

7. Chartrand, G.; Zhang, P. The theory and applications of resolvability in graphs. A Survey. Congr. Numer. 2003, 160, 47-68.

8. Buckley, F. Mean distance in line graphs. Congr. Numer. 1981, 32, 153-162.

9. Cohen, N.; Dimitrov, D.; Krakovski, R.; Skrekovski, R.; Vukasinovic, V. On Wiener index of graphs and their line graphs. MATCH Commun. Math. Comput. Chem. 2010, 64, 683-698.

10. Gutman, I. Distance of line graphs. Graph. Theory Notes N. Y. 1996, 31, 49-52.

11. Gutman, I.; Pavlovic, L. More on distance of line graphs. Graph. Theory Notes N. Y. 1997, 33, $14-18$.

12. Imran, M.; Bokhary, S.A. On resolvability in double-step circulant graphs. UPB Sci. Bull. Ser. A 2014, $76,31-42$.

13. Iranmanesh, A.; Gutman, I.; Khormali, O.; Mahmiani, A. The edge versions of the Wiener index. MATCH Commun. Math. Comput. Chem. 2009, 61, 663-672.

14. Ramane, H.S.; Ganagi, A.B.; Gutman, I. On a conjecture of the diameter of line graphs of graphs of diameter two. Kragujev. J. Math. 2012, 36, 59-62.

15. Ramane, H.S.; Gutman, I. Counterexamples for properties of line graphs of graphs of diameter two. Kragujev. J. Math. 2010, 34, 147-150.

16. Ramane, H.S.; Revankar, D.S.; Gutman, I.; Walikar, H.B. Distance spectra and distance energies of iterated line graphs of regular graphs. Publ. Inst. Math. 2009, 85, 39-46. [CrossRef]

17. Su, G.; Xu, L. Topological indices of the line graph of subdivision graphs and their Schur-bounds. Appl. Math. Comput. 2015, 253, 395-401. [CrossRef]

18. Wu, B. Wiener index of line graphs. MATCH Commun. Math. Comput. Chem. 2010, 64, 699-706.

19. Kelenc, A.; Tratnik, N.; Yero, I.G. Uniquely identifying the edges of a graph: The edge metric dimension. Discret. Appl. Math. 2018. [CrossRef]

20. Nasir, R.; Zafar, S.; Zahid, Z. Edge metric dimension of graphs. Ars Combin. 2018, in press.

21. Feng, M.; Xu, M.; Wang, K. On the metric dimension of line graphs. Discret. Appl. Math. 2013, 161, 802-805. [CrossRef]

22. Cáceres, J.; Hernado, C.; Mora, M.; Pelayo, I.M.; Puertas, M.L.; Seara, C.; Wood, D.R. On the Metric Dimension of Cartesian Products of Graphs. SIAM J. Discret. Math. 2007, 21, 423-441. [CrossRef]

23. Eroh, L.; Kang, C.X.; Yi, E. Metric Dimension and Zero Forcing Number of Two Families of Line Graphs. Math. Biochem. 2014, 139, 467-483.

24. Cangalovic, M.; Kratica, J.; Kovacevic-Vujcic, V.; Stojanovic, M. Minimal doubly resolving sets of prism graphs. Optimization 2013, 62, 1037-1043. [CrossRef]

25. Kratica, J.; Kovacevic, V.; Cangalovic, M.; Stojanovic, M. Minimal doubly resolving sets and the strong metric dimension of Hamming graphs. Appl. Anal. Discret. Math. 2012, 6, 63-71. [CrossRef]

26. Kratica, J.; Kovacevic, V.; Cangalovic, M.; Stojanovic, M. Minimal doubly resolving sets and the strong metric dimension of some convex polytope. Appl. Math. Comput. 2012, 218, 9790-9801. [CrossRef]

27. Ahmad, M.; Zahid, Z.; Zafar, S. On minimal edge version of doubly resolving sets of a graph. arXiv 2018, arXiv:1807.02365.

28. Syslo, M.M.; Proskurowski, A. On Halin graphs. In Graph Theory; Lecture Notes in Mathematics; Springer: New York, NY, USA, 1983; Volume 1018, pp. 248-256.

29. Lovasz, L.; Plummer, M.D. On a family of planar bicritical graphs. Proc. Lond. Math. Soc. 1975, 30, $160-176$. [CrossRef] 
30. Tomescu, I.; Imran, M. R-Sets and Metric Dimension of Necklace Graphs. Appl. Math. Inf. Sci. 2015, 9, 63-67. [CrossRef]

31. Ahmad, A.; Baca, M.; Sultan, S. Minimal Doubly Resolving Sets of Necklace Graph. Math. Rep. 2018, $20,123-129$.

(C) 2018 by the authors. Licensee MDPI, Basel, Switzerland. This article is an open access article distributed under the terms and conditions of the Creative Commons Attribution (CC BY) license (http://creativecommons.org/licenses/by/4.0/). 